\title{
Volumetric Fetal Flow Imaging with Magnetic Resonance Imaging
}

This paper was downloaded from TechRxiv (https://www.techrxiv.org).

\section{LICENSE}

CC BY 4.0

SUBMISSION DATE / POSTED DATE

05-08-2021 / 09-08-2021

CITATION

Goolaub, Datta Singh; Xu, Jiawei; Schrauben, Eric; Marini, Davide; Kingdom, John; Sled, John; et al. (2021): Volumetric Fetal Flow Imaging with Magnetic Resonance Imaging. TechRxiv. Preprint. https://doi.org/10.36227/techrxiv.15112944.v1

$\mathrm{DOI}$

10.36227/techrxiv.15112944.v1 


\title{
Volumetric Fetal Flow Imaging with Magnetic Resonance Imaging
}

\author{
Datta Singh Goolaub, Jiawei Xu, Eric M. Schrauben, Davide Marini, John C. Kingdom, John G. Sled, \\ Mike Seed, and Christopher K. Macgowan
}

\begin{abstract}
Fetal development relies on a complex circulatory network and accurately assessing the flow distribution is important for understanding pathologies and potential therapies. In this paper, we demonstrate a method for volumetric multidimensional imaging of fetal flow with magnetic resonance imaging (MRI). Fetal application of MRI faces several challenges such as small vascular structures, unpredictable motion, and lack of traditional cardiac gating methods. Here, orthogonal multislice stacks are acquired with accelerated multidimensional radial phase contrast (PC) MRI. Each slice is reconstructed into flow sensitive time-series images (CINEs) with retrospective intraslice motion correction and image-based fetal cardiac gating. CINEs are then combined into a dynamic 3D volume using slice-to-volume reconstruction (SVR) while accounting for interslice spatiotemporal coregistration. Validation of the technique is demonstrated in adult volunteers by comparing mean flows from SVR with 4D radial PCMRI with bias and limits of agreement being $-1.1 \mathrm{ml} / \mathrm{s}$ and [-12.5 10.2] $\mathrm{ml} / \mathrm{s}$. Feasibility is demonstrated in late gestation fetuses by comparing SVR with 2D Cartesian PCMRI with bias and limits of agreement being $-0.9 \mathrm{ml} / \mathrm{min} / \mathrm{kg}$ and [-39.7 37.8] $\mathrm{ml} / \mathrm{min} / \mathrm{kg}$ for mean flows. With SVR, we also demonstrate complex flow pathways (such as parallel flow streams in the proximal inferior vena cava, preferential shunting of blood from the ductus venosus into the left side of the heart, and blood returning from the brain leaving the heart through the main pulmonary artery) for the first time in human fetal circulation. This method allows for comprehensive evaluation of the fetal circulation and enables future studies of fetal physiology.
\end{abstract}

Index Terms-4D flow, fetal, flow imaging, MRI, phase contrast.

\section{INTRODUCTION}

$\mathrm{N}$ ormal fetal development relies on an adequate supply of oxygen and nutrients from the placenta to critical fetal organs, such as the fetal myocardium and brain. Delivery is supported by a complex circulatory network that includes physiological shunts such as the ductus venosus, ductus arteriosus, and foramen ovale that are designed to both maximize oxygen transfer to the fetus and to protect oxygen delivery to the fetal brain. These shunts help deliver oxygenated blood from the placenta to the fetal brain and myocardium, with flows partially bypassing the fetal lungs and liver [1]. Fetal pathologies, such as fetal growth restriction and congenital heart disease, disrupt this distribution, causing injury to critical fetal organs and increasing risk of fetal mortality [2], [3]. Accurate measurement of this flow distribution is important because it may identify impairment of organs and thereby help to guide appropriate therapy and monitor efficacy. A complete assessment of the fetal circulatory system is possible through volumetric visualization, which can provide insight into the route of blood in the fetus.

Early studies demonstrated unique fetal flow mechanisms using injection of radiolabeled microspheres [4], [5]. Oxygenated nutrient-rich blood from the placenta partially bypasses the liver through the ductus venosus and flows into the proximal inferior vena cava $\left(\mathrm{IVC}_{\mathrm{p}}\right)$. Upon arrival in the right atrium, this blood takes a preferential route through the foramen ovale into the left atrium, ultimately supplying oxygen and nutrients to the fetal brain and myocardium [5]. Two additional remarkable circulatory adaptations occur in this flow network. First, limited mixing of oxygen-rich blood from the ductus venosus with deoxygenated blood originating from the lower fetal body occurs in the $\mathrm{IVC}_{\mathrm{p}}$ resulting in two parallel streams. Second, deoxygenated blood from the lower and upper fetal body preferentially flows from the right atrium into the right ventricle with limited intracardiac mixing with blood from the ductus venosus. While these mechanisms support normal fetal development, pathologies that disrupt these mechanisms can impair growth and place the fetal brain at risk of hypoxic ischemic injury [2], [6], [7]. Assessment of flow distribution is hence crucial to monitor fetal growth and plan treatment.

Recently, phase contrast magnetic resonance imaging (PCMRI), whereby through-plane velocity is encoded in a single slice prescribed perpendicular to a target vessel, has been investigated for fetal blood flow quantification [8]. However, fetal PCMRI faces several challenges, such as small vasculature structures, high heart rates, uncontrollable motion, lack of traditional cardiac gating methods, and need for expert slice prescription during imaging. Recent advances in accelerated

This work was submitted on 31/07/2021. This work was supported by the Grant PJT148505.

D.S. Goolaub is with Medical Biophysics, University of Toronto and SickKids, Toronto, ON, Canada (e-mail: datta.goolaub@sickkids.ca).

J. Xu is with The Hospital of Sick Children, Toronto, ON, Canada.

E.M. Schrauben was with SickKids, Toronto, ON, Canada. He is now with Amsterdam UMC, location AMC, Amsterdam, NL.

D. Marini is with SickKids, Toronto, ON, Canada.

J.C. Kingdom is with Department of Obstetrics and Gynaecology, Division of Maternal-Fetal Medicine, Sinai Health System, Toronto, Canada

J.G. Sled is with Medical Biophysics, University of Toronto and SickKids, Toronto, ON, Canada.

M. Seed is with Pediatrics, University of Toronto and SickKids, Toronto, Ontario, Canada.

C.K. Macgowan is with Medical Biophysics, University of Toronto and SickKids, Toronto, ON, Canada. 
PCMRI have enabled quantitative assessment of the fetal circulation [2], [9]. These approaches have been used to quantify blood flow in great vessels, allowing estimation of fetal flow maps for healthy and pathological pregnancies [2], [9]. In cases of pathologies, redistribution of fetal flow has been tracked such that development of critical organs can be assessed and patient management can be better planned [2], [10]. However, these studies used single slice measurements with only through-plane velocity encoding. To achieve volumetric flow measurement, multidimensional velocity encoding is needed.

Multidimensional fetal PCMRI has been previously demonstrated using retrospective gating and motion correction [11] using golden-angle radial sampling [12]. While this technique provides high quality fetal images, it is constrained to single-slice use and provides only localized blood flow measurements. One solution to achieve volumetric fetal imaging in the presence of motion involves slice-to-volume reconstruction (SVR) [13]-[15]. This approach, originally developed for fetal brain imaging and later adapted to fetal cardiovascular applications, acquires multiple overlapping stacks of slices which are combined using retrospective 3D motion compensation[12], [13]. Recently, 4D fetal whole heart CINE imaging has been demonstrated using SVR, along with the potential to provide velocity information in the final reconstruction [16], [17].

Here, we present a novel acquisition and reconstruction pipeline for volumetric fetal blood flow visualization and quantification. As explained in the following section, this pipeline relies on SVR to combine multislice radial PCMRI data acquired using an accelerated golden-angle acquisition [11]. Validation is provided in adult volunteers, with feasibility demonstrated in four late gestation human fetuses.

\section{THEORY: ACQUISITION AND RECONSTRUCTION}

\section{A. Acquisition}

Three orthogonal multislice stacks prescribed over a region of interest are acquired using accelerated radial multidimensional PCMRI, which is characterized by three orthogonal velocity measurements in a single slice. As described previously, it involves interleaved velocity encoding directions while continuously updating the trajectory angle by the golden angle [11]. In the stacks, slices have high in-plane resolution with relatively large slice thickness (see Methods for protocol details).

\section{B. Reconstruction}

Reconstruction involves four major steps, as detailed in the subsequent paragraphs and summarized in Fig. 1. First, each slice in each stack is reconstructed independently to yield fetal velocity sensitive CINEs with retrospective motion correction and cardiac gating. Second, the slices are co-registered in space to correct for inter-slice motion. Third, the spatially coregistered slices are temporally synchronized such that the same cardiac phase in all slices occurs simultaneously in the reconstructed dynamic volume. Fourth, the spatiotemporally co-registered slices are reconstructed into a $4 \mathrm{D}$ fetal flow volume.

\section{Step 1: Single slice reconstruction}

In this step, each slice is reconstructed into a velocity sensitive CINE using a previously published framework [11]. The step for reconstructing one slice is explained below and the process is repeated for all slices in all stacks yielding velocity sensitive multislice CINEs. As depicted in Step 1 from Fig. 1, this involves real-time reconstructions for motion correction and cardiac gating followed by velocity sensitive CINE reconstructions. Initial real-time reconstruction is performed for motion correction using a low temporal resolution $(\sim 420 \mathrm{~ms})$ window with compressed sensing (CS) [18]:

$$
\begin{aligned}
\underset{x^{\text {moco }}}{\arg \min } \| F_{s} x^{\text {moco }} & -W^{\text {moco }} y \|_{2} \\
& +\sum_{p} \lambda_{p}\left\|\Psi_{p} x^{\text {moco }}\right\|_{1}
\end{aligned}
$$

where $x^{\text {moco }}$ is the low temporal resolution real-time reconstruction (moco denotes that the reconstruction is used for motion correction), $F_{s}$ is the spatial Fourier operator, $W^{\text {moco }}$ selects temporal windows of spokes in data $y$, and $\Psi_{p}$ are sparsifying transforms with regularizing coefficients $\lambda_{p}$. The sparsifying transforms used in this framework include spatial and temporal total variations (STV and TTV, respectively). Minimization is performed with a conjugate gradient descent algorithm [16].

To correct for in-plane fetal translational motion, real-time frames are co-registered using translational transformations over a user-defined region of interest spanning the fetal heart. Frames sharing low mutual information with other frames (less than the mean by $1.5 \times$ the interquartile range) are defined as through-plane motion and are automatically rejected from the pipeline. The remaining data represent quiescent periods, and the longest period is used for further reconstruction. Registration parameters are interpolated to the repetition time (TR) of the acquisition and applied to correct motion in each spoke retained for reconstruction.

High temporal resolution real-time frames $(\sim 25 \mathrm{~ms})$ are then reconstructed using CS for cardiac gating:

$$
\begin{aligned}
\underset{x^{\text {mog }}}{\arg \min } \| F_{s} x^{\text {mog }} & -W^{\text {mog }} T^{\text {moco }} y \|_{2} \\
& +\sum_{p} \lambda_{p}\left\|\Psi_{p} x^{\text {mog }}\right\|_{1}
\end{aligned}
$$

where $x^{m o g}$ is the high temporal resolution real-time reconstruction, $T^{\text {moco }}$ is the motion correction operator, $W^{\text {mog }}$ creates temporal windows of spokes in acquired data $y$, and sparsifying transforms $\Psi_{p}$ are STV and TTV with regularizing coefficients $\lambda_{p}$. The fetal heart rate is extracted using metric optimized gating (MOG) [19] followed by the data being sorted into cardiac phases.

Finally, multidimensional velocity sensitive CINEs are reconstructed using CS:

$$
\underset{c^{t}}{\arg \min }\left\|F_{S} c^{t}-G T^{\text {moco }} y\right\|_{2}+\sum_{p} \lambda_{p}\left\|\Psi_{p} c^{t}\right\|_{1}
$$

where $c^{t}$ is the multidimensional velocity sensitive CINE, $G$ is a gating operator binning the data $y$ into appropriate cardiac phase $t$, and sparsifying transforms $\Psi_{p}$ are STV and TTV with regularizing coefficients $\lambda_{p}$. Velocity reconstructions are then corrected for background phase errors [20] and phase unwrapped for velocity aliasing. 


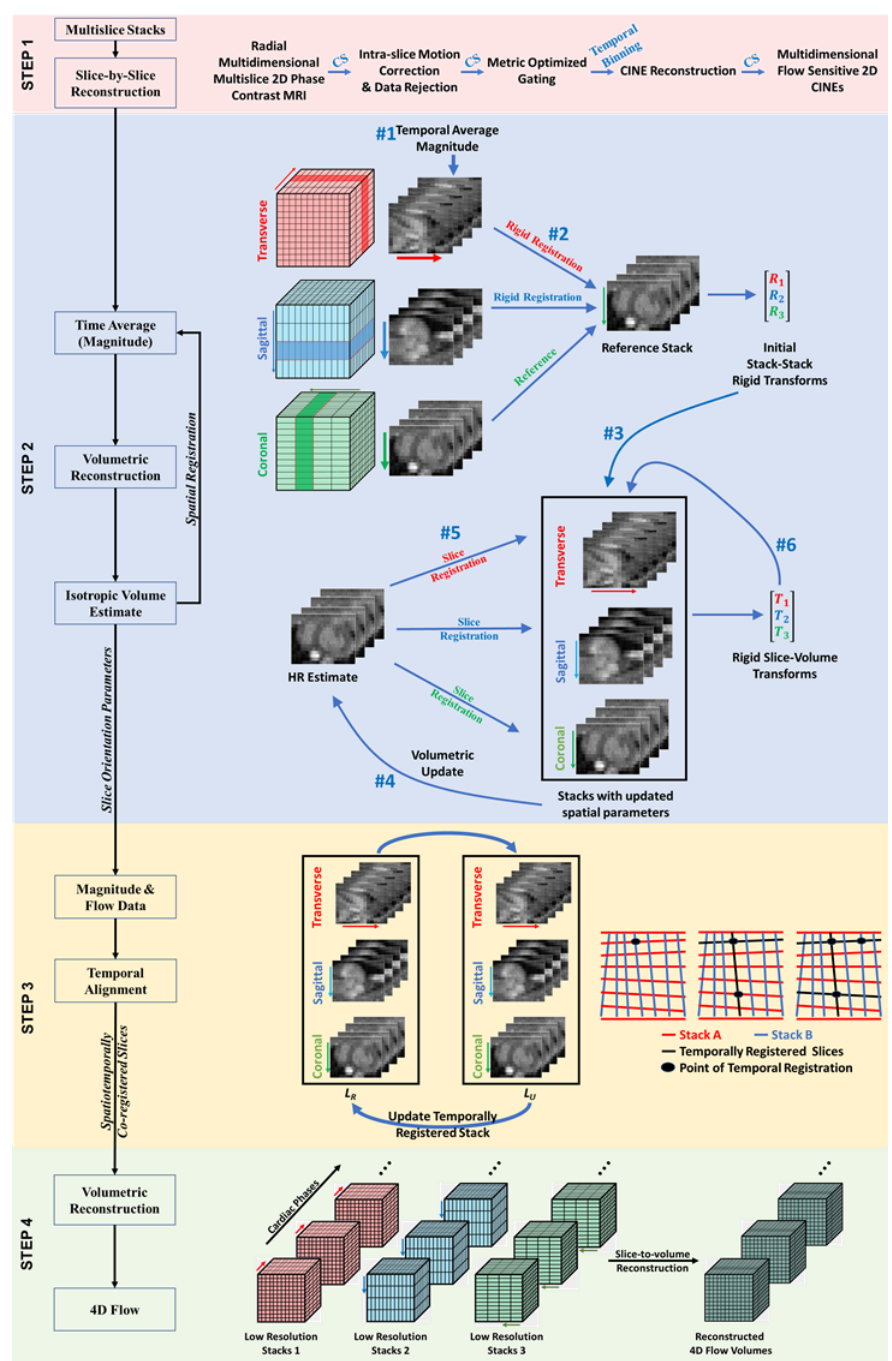

Fig. 1: Pipeline for reconstructing multislice stacks into flow sensitive volumes. Step 1: Reconstruction of slices with multidimensional phase contrast MRI encoding into 2D CINEs with motion correction, metric optimized gating, and compressed sensing (CS). Step 2: Inter-slice motion correction. Stack-to-stack registration correcting for gross fetal motion with stacks of time averaged slices $(\# 1)$ are registered to a reference stack (\#2). Slices are first corrected for stack-to-stack registration (\#3) and then iteratively corrected for finer inter-slice motions. A high resolution (HR) volume is estimated (\#4) and slices are iteratively registered to it (\#5). The registration parameters are used to update the HR volume (\#6). Step 3: Temporal registration of slices. Slices with largest overlap, based on the product of magnitude and speed, are found and registered in the time dimension while updating the temporally registered stack, $L_{R}$, and temporally unregistered stack, $L_{u}$. Step 4: 4D flow reconstruction by applying slice-to-volume reconstruction to the spatiotemporally co-registered flow sensitive slices.

The above steps are repeated for all slices in each stack, $M_{i}$, to yield CINEs, $c_{i}^{k, t}$ (which have an anatomical magnitude component $m_{i}^{k, t}$ and 3 orthogonal velocity components, $v_{i}^{k, t, d}$ with $k$ the slice number, $i$ the stack number, and $d$ the velocity direction).

\section{Step 2: Slice-to-volume registration}

In this step, the reconstructed CINEs are time-averaged and co-registered spatially to each other. The time averaged stack with least inter-slice motion corruption is defined as the reference stack. Rigid registration is first performed to correct for gross motion between the stacks, yielding transforms which align each stack to the reference stack (Fig. 1 Step 2).

A slice-to-volume registration is then performed iteratively to correct for the finer inter-slice motions. Rigid registration is performed to co-register each slice to a time-averaged volume which has been estimated from all the slices using SVR.

In the volumetric reconstruction, slices from different stacks are combined iteratively to generate an isotropic volume. The reconstruction attempts to invert the MRI sampling and motion processes which occurred during the acquisition. SVR is performed by minimizing the following cost function:

$$
\begin{gathered}
\underset{V_{I}}{\arg \min } \sum_{i=1}^{N_{M}} \sum_{k=1}^{N_{i}}\left\|B_{i}^{k} R_{i}^{-1} T_{i}^{k^{-1}} V_{I}-m_{i}^{k}\right\|_{2} \\
+\lambda S T V_{3 D}\left(V_{I}\right)
\end{gathered}
$$

where $V_{I}$ is the volume estimated using SVR, $T_{i}^{k}$ is the rigid registration transform for the $k^{\text {th }}$ slice in the $i^{\text {th }}$ stack, $R_{i}$ is the stack-to-stack registration transform for the $i^{\text {th }}$ stack, $N_{M}$ is the number of acquired stacks, $N_{i}$ is the number of slices in the $i^{\text {th }}$ stack, $B_{i}^{k}$ is the blurring operator based on the point spread function (PSF) of the acquisition process, and $\lambda$ is the weighing coefficient for the 3-dimensional spatial total variation, $S T V_{3 D}$. The PSF of the radial MRI acquisition is jinc $\left(\frac{\sqrt{d x^{2}+d y^{2}}}{\Delta r}\right) \exp \left(\frac{-d z^{2}}{2 \sigma_{z}^{2}}\right)$ where $d x, d y$, and $d z$ are spatial offsets from the reconstructed voxel, $\Delta r$ is the in-plane resolution and $\sigma_{z}$ depends on the prescribed slice thickness. To make use of fast resampling functions, the in-plane PSF is approximated by a Gaussian kernel as in [13].

Slices are registered to $V_{I}$ to yield updated $T_{i}^{k}$ rigid transforms. In this step, the registration solves for 3D translation and 3D rotation parameters which match each slice to its best location in $V_{I}$. The latter are then used to update $V_{I}$. The iterative process, registration followed by SVR, is repeated until the mutual information between the time-averaged volume and the acquired slices converges. In regions of incomplete slice coverage due to high fetal motion, interslice spatial interpolation is performed as part of the SVR. The final rigid transforms $T_{i}^{k}$ at this stage provide spatial co-registration of all $c_{i}^{k, t}$ slices, with corresponding velocity measurements $v_{i}^{k, t, d}$ reoriented accordingly.

\section{Step 3: Temporal Synchronization of CINEs}

The spatially co-registered dynamic slices, $c_{i}^{k, t}$, must next be synchronized in time. To address this, temporal synchronization is performed across all slices based on their regions of overlap. Synchronization is performed using the product of image magnitude and speed, $E_{i}^{k, t}$ given by $m_{i}^{k, t} \sqrt{\sum_{d}\left[v_{i}^{k, t, d}\right]^{2}}$. Strength of overlap between regions is first computed. An adjacency matrix for all slice pairs is populated using the sum of $E_{i}^{k, t}$ in their region of overlap. A weighted graph is then computed representing the connections between slices [21]. The slice with the strongest connections is used as the seed point for the synchronization. As in Step 3 in Fig. 1, synchronization is performed iteratively by: (1) choosing a synchronized and unsynchronized slice with strongest overlap, (2) temporally shifting the unsynchronized slice until the 
entropy over the region of overlap is minimized, (3) looping until all slices are synchronized.

\section{Step 4: 4D velocity reconstruction}

With the slices, $c_{i}^{k, t}$, spatiotemporally co-registered, the slices are intensity corrected as in [14]. Scaling factors are computed between lowpass filtered slices in the reference stack and those from the other stacks. Intensity correction minimizes bias caused by anatomical magnitude disparities during combination of velocity sensitive complex data. The final 4D flow reconstruction (Step 4 in Fig. 1) is then performed by minimizing the cost function given by:

$$
\begin{gathered}
\underset{V_{I}^{t}}{\arg \min } \sum_{i=1}^{N_{M}} \sum_{k=1}^{N_{i}}\left\|B_{i}^{k} R_{i}^{-1} T_{i}^{k^{-1}} V_{I}^{t}-c_{i}^{k, t}\right\|_{2} \\
+\lambda T V\left(V_{I}^{t}\right)
\end{gathered}
$$

The reconstructed volume $V_{I}^{t}$ is a $4 \mathrm{D}$ volume containing dynamic volumetric magnitude and multidimensional velocity data. In this manuscript, 4D flow reconstruction generated using this approach will henceforth be referred to as 'radial SVR flow'.

\section{METHODS}

\section{A. Experimental Validation in Adults}

To validate the framework, two adult volunteers (A1, A2; 25 years) were recruited with informed consent and scanned using a commercial 3T system (Prisma ${ }^{\mathrm{FIT}}$, Siemens Healthineers, Erlangen, Germany). Scanning protocol involved 3 orthogonal stacks of multislice 2D multidimensional radial PCMRI followed by a previously described 4D radial flow MRI acquisition [22]. This protocol was repeated 3 times in each adult. Scans were performed under free breathing conditions with the respiratory bellows and pulse oximeter gating physiological logs recorded. The prescribed volume focused on the main pulmonary artery, the aortic arch, and the descending aorta for radial SVR flow, and the whole chest for 4D radial flow. Relevant imaging parameters, chosen to keep scan times approximately equal between the two acquisition approaches, are summarized in Table I.

\section{B. Fetal scans}

Four pregnant women with healthy singleton fetuses (gestation: $36 \pm 1$ weeks, subject identifiers: F1 - F5) were scanned with informed consent using the same commercial $3 \mathrm{~T}$ system. For reference flow measurements, 2D Cartesian PCMRI acquisitions were performed on the major fetal vessels (DAO: descending aorta, AAO: ascending aorta, SVC: superior vena cava, MPA: main pulmonary artery, DA: ductus arteriosus) [19]. Acquisitions were repeated if an initial reconstruction on the scanner was deemed to be motion corrupted. Three orthogonal stacks of multislice multidimensional 2D radial PCMRI, centered on the fetal heart, were also acquired. Relevant imaging parameters are summarized in Table II. 500 radial spokes per flow encode were acquired. This acquisition represented an acceleration factor (R) of 12. Previous studies showed that radial acquisitions in human fetuses were accurately reconstructed with $\mathrm{R} \sim 18$ using CS [11], [23]. Hence, around $33 \%$ of the data from the
TABLE I

ACQUISITION PARAMETERS FOR ADULT 4D FLOW AND RADIAL PHASE CONTRAST MRI SCANS. TE: ECHO TIME, TR: REPETITION TIME, AND R: ACCELERATION FACTOR

Phase Contrast MRI Protocol 4D Radial Flow Radial SVR Flow

\begin{tabular}{ccc}
\hline Scan Time & 7.2 mins & $\begin{array}{c}4.5 \text { mins } \\
(5.5 \text { s per slice })\end{array}$ \\
\hline $\begin{array}{c}\text { Voxel Size } \\
\left(\mathrm{mm}^{3}\right)\end{array}$ & $2 \times 2 \times 2$ & $2 \times 2 \times 8$ \\
\hline TE / TR $(\mathrm{ms})$ & $3.0 / 5.4$ & $3.6 / 6.1$ \\
\hline Flip Angle $\left(^{\circ}\right)$ & 8 & 25 \\
\hline $\mathrm{R}$ & $\sim 30$ & $\sim 13$ \\
\hline $\begin{array}{c}\text { Spokes per } \\
\text { encode }\end{array}$ & $\begin{array}{c}\sim 14000 \text { with } \\
\text { respiratory gating }\end{array}$ & 225 \\
\hline $\begin{array}{c}\text { Encode Vel. } \\
(\mathrm{cm} / \mathrm{s})\end{array}$ & 150 & 150 \\
\hline $\begin{array}{c}\text { Base } \\
\text { Resolution }\end{array}$ & $128 \times 128 \times 128$ & $128 \times 128$ \\
\hline Slices / Stacks & - & $\sim 15 / 3$ \\
\hline \hline
\end{tabular}

acquisitions could be rejected during motion correction while still allowing for CINE reconstruction.

\section{Adult Flow Reconstructions}

The acquired 4D radial flow data were first binned into 3 respiratory phases, based on the recorded respiratory log. The end expiratory phase was then binned into 15 cardiac phases, based on the pulse oximeter gating log, and reconstructed using $\mathrm{CS}(\mathrm{R} \sim 30)$ with spatial total variation $(\mathrm{STV} ; \lambda=0.008)$ and temporal total variation (TTV; $\lambda=0.01)$ regularization.

The multislice acquisitions were reconstructed as described above and in [11], but without intra-slice motion correction since acquisitions were short and initial real-time reconstructions showed negligible motion of the aorta and main pulmonary artery. Multidimensional velocity sensitive CINEs were then reconstructed using $\operatorname{CS}(\mathrm{R} \sim 13)$ with $\operatorname{STV}(\lambda=0.001)$ and TTV $(\lambda=0.008)$ regularization. Slices were then passed into the volumetric reconstruction pipeline, with inter-slice motion correction and temporal alignment. Final velocity volumetric reconstructions were performed with $\operatorname{STV}(\lambda=0.01)$ regularization.

TABLE II

ACQUISITION PARAMETERS FOR CARTESIAN AND RADIAL PHASE CONTRAST MRI FETAL SCANS. TE: ECHO TIME, TR: REPETITION TIME, AND R: ACCELERATION FACTOR

Phase Contrast MRI Protocol 2D Cartesian Radial SVR Flow

\begin{tabular}{ccc}
\hline Scan Time & $22 \mathrm{~s}$ & $\begin{array}{c}6.5 \mathrm{mins} \\
(13 \mathrm{~s} \text { per slice })\end{array}$ \\
\hline $\begin{array}{c}\text { Voxel Size } \\
\left(\mathrm{mm}^{3}\right)\end{array}$ & $1.3 \times 1.3 \times 5.0$ & $1 \times 1 \times 4$ \\
\hline TE / TR $(\mathrm{ms})$ & $3.0 / 6.8$ & $3.9 / 6.6$ \\
\hline Flip Angle $\left(^{\circ}\right)$ & 20 & 25 \\
\hline $\mathrm{R}$ & 2 & $\geq 12$ \\
\hline $\begin{array}{c}\text { Encode Vel. } \\
(\mathrm{cm} / \mathrm{s})\end{array}$ & $100-150$ & 100 \\
\hline $\begin{array}{c}\text { Base } \\
\text { Resolution }\end{array}$ & $192 \times 192$ & $\begin{array}{c}256 \times 256 \\
(500 \text { spokes per encode })\end{array}$ \\
\hline Slices / Stacks & $1 / 1$ & $\sim 10 / 3$ \\
\hline \hline
\end{tabular}




\section{Fetal Flow Reconstructions}

2D Cartesian PCMRI acquisitions were reconstructed into CINEs, with 15 cardiac phases, using MOG as previously described [8], [19]. The multislice radial acquisitions were reconstructed as described above and in [11]. Initial real-time reconstructions for motion correction were performed with CS using $\operatorname{STV}(\lambda=0.01)$ and $\operatorname{TTV}(\lambda=0.001)$ regularization. Motion corrected data were reconstructed into high temporal resolution real-time images for cardiac gating with $\mathrm{CS}$ using $\operatorname{STV}(\lambda=0.01)$ and TTV $(\lambda=0.001)$ regularization. The fetal heart rate was extracted using MOG followed by binning the data into 15 cardiac phases. Velocity sensitive CINEs were reconstructed with $\operatorname{CS}(\mathrm{R} \geq 12)$ using STV $(\lambda=0.01)$ and TTV $(\lambda=0.05)$ regularization. Slices were then passed into the volumetric reconstruction pipeline for inter-slice motion correction and temporal alignment. Final velocity volumetric reconstructions were performed using STV $(\lambda=0.01)$ regularization. In regions of incomplete slice coverage due to high fetal motion, spatial interpolation was performed as part of the SVR.

\section{E. Analysis adult}

All flow analysis and visualizations were performed using a prototype software (4D Flow v2.4, Siemens) [24]. The heart and great vessels were first segmented by thresholding the 4D flow data based on peak velocities, to create a phase-contrast angiogram. Refined semi-automated segmentation was then initiated through user-selected seed points for vessel centerline and lumen extraction. Mean flows were recorded at similar positions along the aorta and main pulmonary artery in the 4D flow and the radial SVR flow reconstructions. The flow values were compared using a paired t-test (with significance level set at $p<0.05$ ), linear regression, and Bland-Altman analysis (with the difference computed by subtracting the radial SVR flow from the 4D flow measurements).

\section{F. Analysis fetal}

2D Cartesian reconstructions were analyzed using the commercially available software QFlow ${ }^{\circledR}$ from Medis Suite MR (Medis Medical Imaging, Leiden, The Netherlands).

From the reconstruction pipeline for the radial MRI data, the mean and range of the displacements and the percentage of data rejected owing to through-plane motion during intra-slice registration were recorded. The mean and range of the displacements and rotational Euler angles were also tracked for inter-slice registration. The mean and the standard deviation of the fetal heart rate obtained from MOG were recorded.

All volumetric flow analysis and visualizations of radial SVR flow data were performed using the prototype software as before. For flow measurements, contours were initially placed at 3 locations along each visible great vessel. The contours were refined by reorienting them perpendicular to the vessel axis and the flows through the cross sections were measured. The mean flows, indexed to fetal mass [8], from the volumetric and Cartesian reconstructions were compared by paired t-test, linear regression, and Bland-Altman analysis (with the difference computed from Cartesian PCMRI measurements subtracted from radial SVR flow). Internal consistency checks in the volumetric reconstruction were performed by assessing the conservation of mass in the reconstructed circulatory network. The flow in the DAO was compared with the incoming flows from the DA and the aortic isthmus (AI) through DAO = AI + DA. The flow in the SVC was compared with the flow leaving the aortic arch through $\mathrm{SVC}=\mathrm{AAO}-\mathrm{AI}$. The flow entering the heart from the SVC, IVC (which is assumed to be equal to DAO flow), and the pulmonary veins (PV, given by the difference between the MPA and DA flows) were compared with the combined ventricular output $(\mathrm{CVO}=1.03 \times[\mathrm{MPA}+\mathrm{AAO}])$ [1]. The consistencies were computed as a percentage of the CVO.

\section{G. Fetal Flow Visualization}

Radial SVR flow allowed for various ways to visualize the fetal circulation. Representative examples, depicting volumetric flow distribution, from the four fetal cases were reported here. To visualize fetal blood flow, particles were seeded throughout the segmentations in subject F1 and F2. Path lines were generated to visualize the movement of blood in the fetal heart and surrounding great vessels. To track fetal blood flow originating from specific locations, particle tracing was performed by placing emitter planes in target fetal vessels. In subject F3, emitter planes were placed in the ductus venosus and IVC to visualize the paths of oxygenated blood coming from placenta and deoxygenated blood coming from the lower fetal body. In subject F4, emitter planes were placed in the SVC and $\mathrm{IVC}_{\mathrm{p}}$ to look at the path of blood in the right side of the heart as it arrives from the fetal upper and lower body.

\section{H. Reconstruction Specifications}

All reconstructions in this work were performed on a standard desktop computer with the following specifications: 32 GB memory, processor Intel Core i7-6700 $(3.40 \mathrm{GHz}, 8$ cores) and GPU Nvidia Geforce GTX 960 (2GB and 1024 CUDA cores). Regularization coefficients $(\lambda)$ were optimized through empirical testing. For 4D radial flow, values for $\lambda$ were chosen such that reconstructed flow values were internally consistent. Values of $\lambda$ for CS were chosen such that the undersampled reconstructions were consistent with highly sampled reconstructions in prior tests. Since the acquisition of highly sampled dataset for SVR is impractical in-vivo, $\lambda$ for SVR was solved using a grid search through simulated data by finding the $\lambda$ that yielded a reconstructed $3 \mathrm{D}$ phantom with lowest errors from simulated slices.

\section{RESULTS}

\section{A. Adult Flow Comparisons}

4D radial flow reconstructions in adults required approximately 3 hours. Radial SVR flow reconstructions required approximately 5 hours.

Representative reconstructed CINE frames from one subject (A1) are presented in Fig. 2A, showing anatomy and flow in peak systole from each orthogonal stack. Subsequent SVR reconstruction based on multislice CINE data found that interslice displacements and rotations were small across all slices from both volunteers (displacements of $1.4 \pm 1.1 \mathrm{~mm}$ and rotational Euler angles of $\mathrm{E}_{\mathrm{x}}=-0.1^{\circ} \pm 0.2^{\circ}, \mathrm{E}_{\mathrm{y}}=0.0^{\circ} \pm 0.2^{\circ}, \mathrm{E}_{\mathrm{z}}$ $=0.0^{\circ} \pm 0.1^{\circ}$ ). Fig. $2 \mathrm{~B}$ shows a rendering of the $3 \mathrm{D}$ cardiac anatomy from A1 reconstructed with SVR. Oblique resampled 
slices, from the reconstructed volume, depicting the surrounding great vessels near the heart are shown in Fig. 2C. Particle traces tracking blood flow in the aorta and MPA evolving over a systolic phase are shown in Fig. 2D. Blood can be seen going into the left pulmonary artery from the MPA and down the DAO from the AAO. Comparison of mean flows between the radial SVR flow and 4D radial flow reconstructions is shown in Fig. 3. Linear regression (Fig. 3A) showed good agreement between the two techniques with slope $=0.99(95 \%$ confidence interval $\left.=\left[\begin{array}{ll}0.93 & 1.07\end{array}\right]\right)$, intercept $=1.27 \mathrm{ml} / \mathrm{s}(95 \%$ confidence interval $=[-4.276 .81] \mathrm{ml} / \mathrm{s})$, and $\mathrm{r}^{2}=0.92$. BlandAltman analysis (Fig. 3B) had a bias of $-1.1 \mathrm{ml} / \mathrm{s}$ with limits of agreement $[-12.510 .2] \mathrm{ml} / \mathrm{s}$. Paired t-test showed that there was no significant difference between the measurements $(p=0.11)$.

\section{B. Fetal Flow Comparisons}

Reconstruction of each slice from the multidimensional radial phase contrast MRI in the fetus required around 45 mins and the SVR required approximately 4 hours. For intra-slice motion correction, the median of the absolute displacement was $1.0 \mathrm{~mm}$ with an interquartile range of $\left[\begin{array}{ll}0.3 & 1.1\end{array}\right] \mathrm{mm}$. The maximum intra-slice displacement occurred in F2 and had a



Fig. 2: Representative slices from orthogonal stacks in adult, A1. (A) Axial, coronal, and sagittal slices show the magnitude and velocity sensitive reconstructions. (B) 3D rendering of reconstructed anatomy from slice-to-volume reconstruction (SVR). (C) Oblique slices from reconstructed volume using SVR. The colored asterisks ( $\left.{ }^{*}\right)$ denote the slice position in the volume. (D) Left lateral view of systolic particle tracking shown in the MPA and AAO from SVR flow.
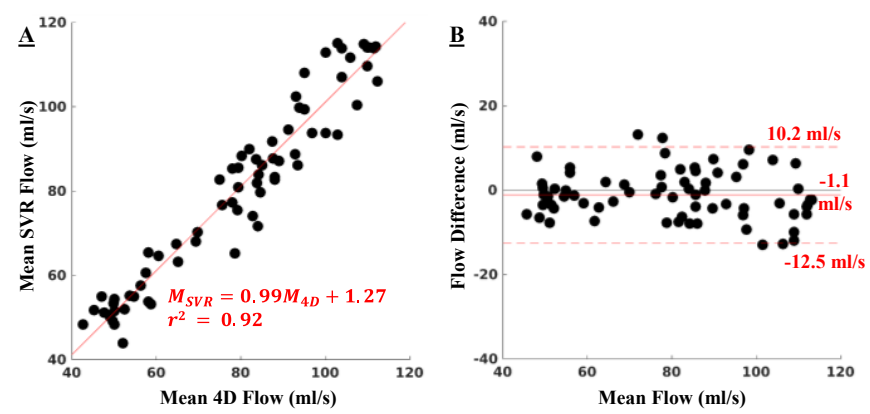

Fig. 3: Comparison between mean flow measurements from slice-tovolume reconstruction and $4 \mathrm{D}$ flow in the aorta and main pulmonary artery of two adult volunteers. (A) Linear regression with red line depicting the best fit to the data. (B) Bland-Altman plot comparing the mean flows. The difference is computed by subtracting the radial SVR flow from the 4D flow measurements. The solid red line depicts the bias, and the dashed red lines depict the upper and lower limits of agreement.

magnitude of $21.2 \mathrm{~mm}$. To discard through-plane motion, the median percentage of data rejected in slices was $0 \%$ with an interquartile range of [ $\left.\begin{array}{ll}0 & 3.6\end{array}\right] \%$. The largest portion of data for one slice $(29 \%)$ was rejected in the axial stack of F2. The percentage of raw data rejected was generally high in this stack with a mean of $10 \%$ and interquartile range of [2 21] \%. The cardiac cycle duration across all fetal subjects was $430 \pm 25 \mathrm{~ms}$. Supplementary File 1 summarizes the motion estimates and heart rate for the fetal data calculated by the reconstruction pipeline.

Representative reconstructed CINEs from one fetus (F3) are shown in Fig. 4A. The anatomy and velocity reconstructions at systole are presented for fetal axial, coronal, and sagittal planes. From SVR based on the CINE data, the average inter-slice displacement was $5.6 \pm 4.6 \mathrm{~mm}$ for all slices across all subjects. The corresponding average rotational Euler angles were $\mathrm{E}_{\mathrm{x}}$ $=-0.4^{\circ} \pm 0.9^{\circ}, \mathrm{E}_{\mathrm{y}}=-0.4^{\circ} \pm 0.6^{\circ}, \mathrm{E}_{\mathrm{z}}=-0.1^{\circ} \pm 0.7^{\circ}$. Largest sliceto-volume displacements were observed in F3 $(7.7 \pm 6.8 \mathrm{~mm}$, maximum: $18 \mathrm{~mm}$, angles [minimum maximum]: $\mathrm{E}_{\mathrm{x}}=\left[-2.0^{\circ}\right.$ $\left.\left.2.9^{\circ}\right], \mathrm{E}_{\mathrm{y}}=\left[\begin{array}{ll}-2.5^{\circ} 0.5^{\circ}\end{array}\right], \mathrm{E}_{\mathrm{z}}=\left[\begin{array}{ll}-2.2^{\circ} & 1.2^{\circ}\end{array}\right]\right)$. Fig. 4B shows a rendering of the $3 \mathrm{D}$ cardiac anatomy and surrounding great vessels in the F3 reconstructed with SVR. Oblique resampled slices, from the reconstructed volume, are shown in Fig. 4C. One visualization result of the SVR is shown in Fig. 4D using particle traces to track the ejection of blood from the ventricles into the aorta and MPA over a systolic phase. Blood can be seen going from the right ventricle into the MPA and from the left ventricle into the AAO.

Fetal flow waveforms obtained using radial SVR flow showed good agreement with 2D Cartesian PCMRI measurements, as demonstrated by the representative curves from one fetus (F3) shown in Fig. 5. Features of these waveforms follow typical fetal arterial and venous patterns, and are consistent with prior publications: for instance the biphasic pulsatile behavior in the SVC can be observed [8], [25].

Fetal mean flows measured using radial SVR flow compared well with those obtained from target vessels using 2D Cartesian PCMRI. As shown in Fig. 6A, linear regression between flows across all vessels and fetuses had a slope $=0.94(95 \%$ confidence interval $\left.=\left[\begin{array}{ll}0.72 & 1.16\end{array}\right]\right)$, intercept $=8.9(95 \%$ confidence interval $=[-29.347 .2])$, and $\mathrm{r}^{2}=0.86$. Bland-Altman analysis (Fig. 6B) had a bias of $-0.9 \mathrm{ml} / \mathrm{min} / \mathrm{kg}$ and limits of 


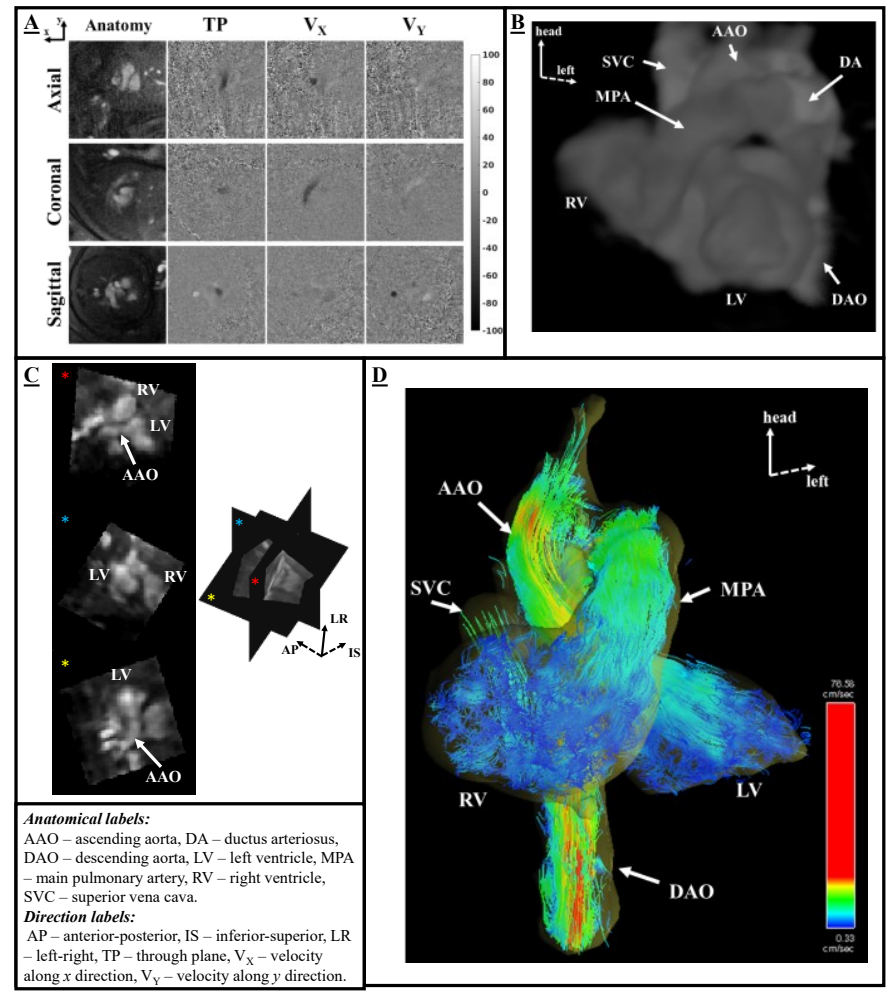

Fig. 4: Representative slices from orthogonal stacks in fetus, F3. (A) Axial, coronal, and sagittal slices show the magnitude and velocity sensitive reconstructions. (B) 3D rendering of reconstructed anatomy from slice-to-volume reconstruction (SVR). (C) Oblique slices from reconstructed volume using SVR. The colored asterisks $\left({ }^{*}\right)$ denote the slice position in the volume. (D) Coronal view of systolic particle tracking show ejection of blood into the MPA and AAO.

agreement [-39.7 37.8] $\mathrm{ml} / \mathrm{min} / \mathrm{kg}$. Paired t-test showed that there was no significant difference between the measurements $(p=0.86)$. The CVO across the subjects was $385 \pm 41$ $\mathrm{ml} / \mathrm{min} / \mathrm{kg}$ from the radial SVR. The corresponding measurement from the 2D Cartesian PCMRI was $375 \pm 46$ $\mathrm{ml} / \mathrm{min} / \mathrm{kg}$. Table III compares the mean flows and ranges of flows in fetal great vessels from literature [9], with Cartesian PCMRI and radial SVR flow. The measured flows in all the categories fall in the same range. Conservation of mass checks across the various cardiac structures showed differences of $7 \pm$ 9\%. Proximal to the aortic isthmus, the percentage difference between DAO and AI + DA flows, relative to CVO, was $8 \pm$ $5 \%$. The percentage difference between the SVC and the flow leaving the aorta for the upper body was $11 \pm 8 \%$. The
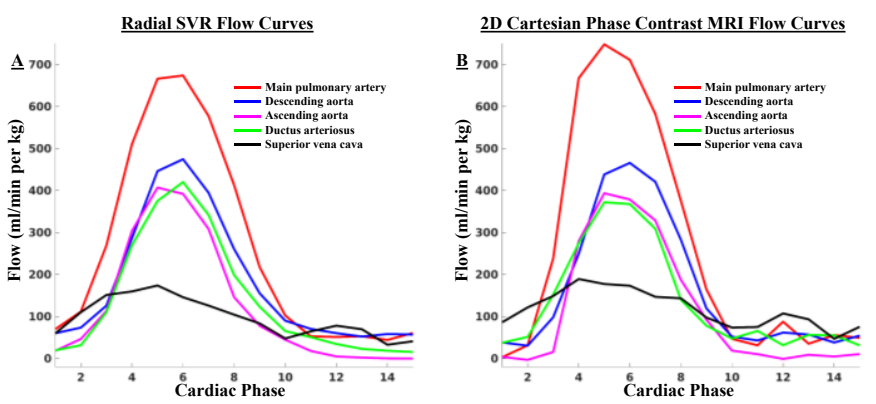

Fig. 5: Flow curves in fetal great vessels. A: Radial slice-to-volume reconstruction (SVR) flow measurements. B: Cartesian phase contrast MRI flow measurements.
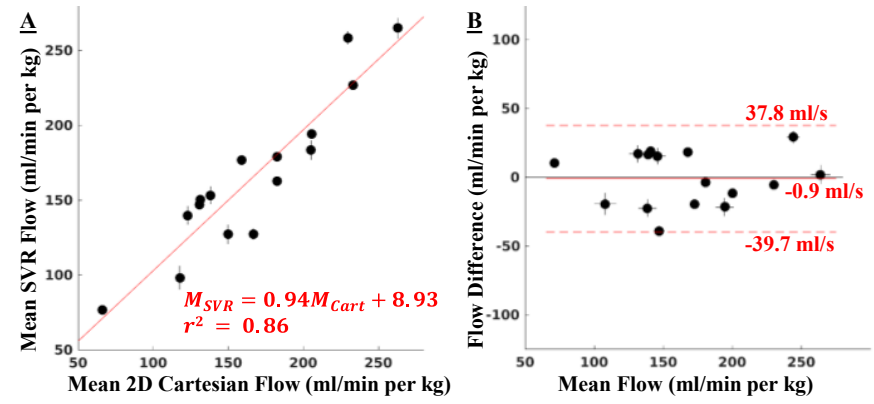

Fig. 6: Comparison between mean flow measurements from radial slice-to-volume flow reconstruction and through-plane Cartesian phase contrast measurements in fetal great vessels. (A) Linear regression for mean flows. Red line depicts the fitted line to the data. (B) Bland-Altman plot comparing the mean flows. The flow difference is obtained by subtracting Cartesian phase contrast measurements from the radial slice-to-volume flow measurements. The solid red line depicts the bias, and the dashed red lines depict the upper and lower limits of agreement. The error bars denote the standard deviation in the measurements obtained at different locations in each great vessel in the slice-to-volume reconstructions.

difference between the CVO and the blood entering the heart was $2 \pm 14 \%$.

\section{Fetal Flow Visualization}

Particle traces tracking fetal blood flow in the heart and outflow tracts are depicted in Fig. 7A for F1 and Fig. 7B for F2. Ejection of the blood from the right ventricle into the MPA and the left ventricle into the AAO are depicted in Fig. 7A1 and Fig. 7B1. Blood flow from the SVC into the heart can also be observed in Fig. 7A1. Blood moving into the ventricle during diastole can be observed in Fig. 7A2 and Fig. 7B2. Supplementary File 2 shows a movie for the flow in F2 at different cardiac phases.

Particle traces depicting flow in the proximal IVC are shown in Fig. 8A with planes set in the ductus venosus (red) and distal IVC (blue) simultaneously emitting particles in F3. The figure demonstrates the path of blood over two heart beats. While both streams meet in the proximal IVC, the blood streams from the two vessels have limited mixing (Fig. 8A1). Blood from the ductus venosus preferentially shunts to the left side of the heart and most of the blood from the distal IVC goes to the right side of the heart (Fig. 8A2). Blood that originated from the ductus venosus or distal IVC exits the heart through the AAO or MPA respectively (Fig. 8A3). Fig. 8B depicts particle traces of flow from SVC and proximal IVC in F4. Simultaneous emission of particles from planes in the SVC (blue) and proximal IVC (red) are shown in Fig. 8B1. SVC blood flows into the right side of the heart while that from the IVC flows into both sides of the heart (Fig. 8B2). Traces in the AAO show that blood originated only from the IVC while those in the MPA show that the flowing blood originated from both the SVC and IVC. Supplementary File 3 shows a movie for the tracked flow paths in F3 and F4 at different cardiac phases.

\section{DISCUSSION}

In this study, we devised an approach using rapid multislice multidimensional radial PCMRI to image the volumetric human fetal blood flow. Reconstructions were performed using CS with cardiac gating through MOG, and retrospective intra-slice and inter-slice motion correction. Radial SVR flow was tested 


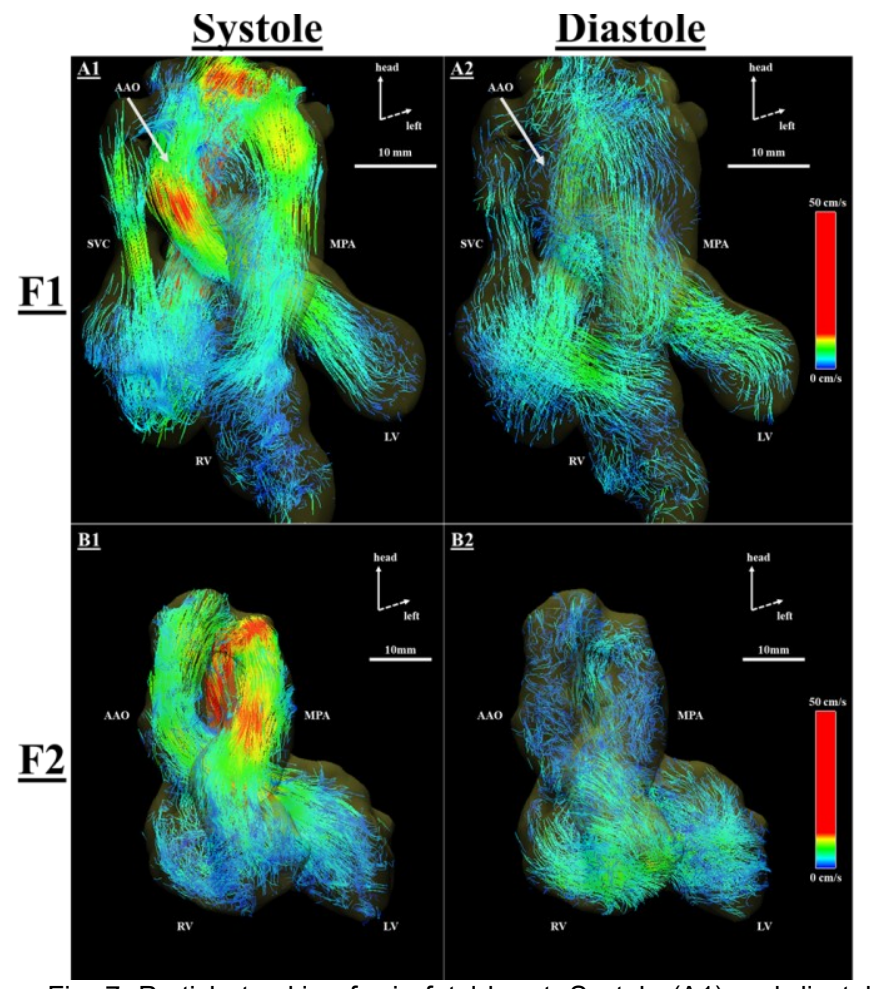

Fig. 7: Particle tracking for in fetal heart. Systole (A1) and diastole (A2) in subject F1 in coronal view. Systole (B1) and diastole (B2) in subject $F 2$. Ventricular output is observed in $A 1$ and $B 1$. Ventricle filling is observed in A2 and B2. AAO - ascending aorta, LV - left ventricle, MPA - main pulmonary artery, RV - right ventricle, SVC - superior vena

in adult subjects and compared with $4 \mathrm{D}$ radial flow MRI measurements. Feasibility of the approach was demonstrated in four human fetuses in the late third trimester and compared with 2D Cartesian PCMRI measurements in the great vessels. Along with fetal blood flow quantification, we were able to visualize complex fetal hemodynamics. We showed parallel streams of blood in the IVC and preferential blood flow routes in the fetal heart.

In the experiments in adults, radial SVR flow showed good agreement with $4 \mathrm{D}$ radial flow reconstructions. Since radial SVR flow was based on 2D slice acquisitions, it benefited from
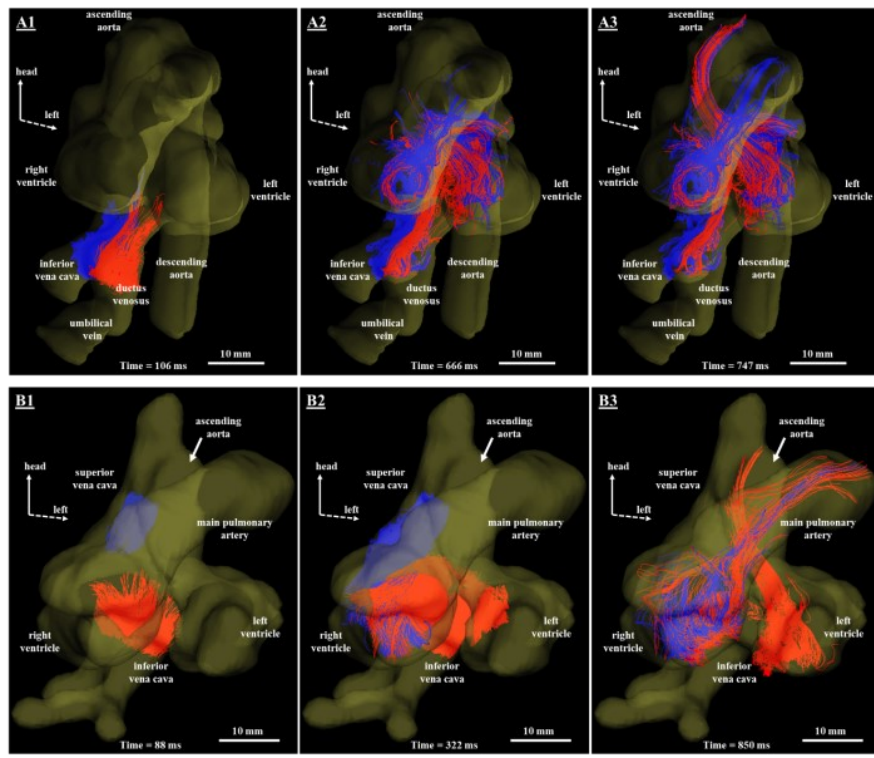

Fig. 8: Tracking flow from specific locations in fetal subjects. Particle tracking in a coronal oblique view in $\mathrm{F} 3$ (A) and top-down oblique view in F4 (B). (A1) Blood flowing from inferior vena cava (blue) and ductus venosus (red) in parallel streams towards the heart. (A2) Blood from ductus venosus preferentially goes to the left ventricle while that from inferior vena cava goes mainly in the right ventricle. (A3) Ejection of blood during systole with blood originating from ductus venosus going in the ascending aorta and that originating from the inferior vena cava moves through the main pulmonary artery. (B1) Blood flowing from the superior vena cava (blue) and proximal inferior vena cava (red). (B2) Stream from the superior vena cava stays in the right side of the heart while blood from the proximal inferior vena cava moves into both sides of the heart. (B3) Ejection of blood during systole with blood originating from the superior vena cava going through the main pulmonary artery this blood does not return to the brain before reoxygenation.

in-flow related enhancement with saturated spins being washed out of the imaged region and fresh spins moving in. This allowed the blood vessels to have higher intensities and become more conspicuous in the SVRs.

To achieve good contrast in conventional clinical 4D flow MRI, contrast agents (such as ferumoxytol and gadolinium) are injected [26]. However, contrast agents have disadvantages. In addition to their expense, injection of the contrast agent into the

TABLE III

COMPARISON OF MEAN, STANDARD DEVIATION (SD) AND RANGE OF FETAL FLOWS IN THE GREAT VESSELS FROM LITERATURE, 2D CARTESIAN PHASE CONTRAST MRI AND RADIAL SVR FLOW

\begin{tabular}{|c|c|c|c|c|c|c|c|}
\hline & & CVO & MPA & 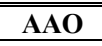 & SVC & DA & $\overline{\text { DAO }}$ \\
\hline \multirow{4}{*}{ Literature Flow Values } & $\begin{array}{c}\text { Mean Flow } \\
\mathrm{ml} / \mathrm{min} \text { per } \mathrm{kg}\end{array}$ & 465 & 261 & 191 & 137 & 187 & 252 \\
\hline & SD & 57 & 46 & 35 & 30 & 39 & 46 \\
\hline & $\begin{array}{c}\text { Range } \\
\mathrm{ml} / \mathrm{min} \text { per kg }\end{array}$ & 351,579 & 169,353 & 121,261 & 77,197 & 109,265 & 160,344 \\
\hline & $\% \mathrm{CVO}$ & - & 56 & 41 & 29 & 40 & 55 \\
\hline \multirow{4}{*}{$\begin{array}{c}\text { 2D Cartesian } \\
\text { Phase Contrast MRI }\end{array}$} & $\begin{array}{c}\text { Mean Flow } \\
\mathrm{ml} / \mathrm{min} \text { per } \mathrm{kg}\end{array}$ & 376 & 225 & 140 & 111 & 150 & 200 \\
\hline & SD & 46 & 40 & 23 & 42 & 28 & 31 \\
\hline & $\begin{array}{c}\text { Range } \\
\mathrm{ml} / \mathrm{min} \text { per } \mathrm{kg}\end{array}$ & 338,428 & 177,271 & 119,151 & 74,132 & 145,164 & 175,229 \\
\hline & $\%$ CVO & - & 60 & 37 & 29 & 40 & 53 \\
\hline \multirow{4}{*}{ Radial SVR Flow } & $\begin{array}{c}\text { Mean Flow } \\
\mathrm{ml} / \mathrm{min} \text { per } \mathrm{kg}\end{array}$ & 384 & 234 & 139 & 101 & 154 & 195 \\
\hline & SD & 41 & 48 & 11 & 26 & 8 & 22 \\
\hline & $\begin{array}{c}\text { Range } \\
\mathrm{ml} / \mathrm{min} \text { per kg }\end{array}$ & 339,417 & 179,265 & 127,150 & 77,129 & 147,162 & 177,227 \\
\hline & $\% \mathrm{CVO}$ & - & 60 & 36 & 26 & 40 & 52 \\
\hline
\end{tabular}

Literature-based numbers are from [9] 
fetal circulation is challenging owing to potential injuries in case of fetal motion and difficulty in localizing the site of injection. Moreover, ferumoxytol can lead to hypertension and gadolinium has been linked to nephrogenic systemic fibrosis in patients with kidney failure [27], [28]. With such drawbacks, contrast agents cannot be administered to fetal subjects and the flow enhancements in the SVR approach provide a fitting tool for imaging the intrauterine circulatory system.

The range of flow values observed in this study using radial SVR flow agreed well to that obtained using Cartesian PCMRI and reference normal fetal blood flow measurements reported in literature [8], [9]. Furthermore, the values for internal consistency based on conservation of mass were comparable to internal consistency checks performed with 4D flow in animal fetuses [29]-[31]. These observations build confidence in the conservation of mass checks in this study. Also, it is important to note that the Cartesian scans were repeatedly acquired until an initial reconstruction on the scanner did not show signs of motion corruption. On average, Cartesian flow measurements were acquired twice, with localizer scans also rerun for prescription. Consequently, the total imaging time of the Cartesian scans ( 10 mins) was longer than the imaging time of the radial SVR flow ( $\sim 6.5$ mins) scans.

In addition to the quantitative agreements, the volumetric visualizations corroborate previously demonstrated circulatory physiological mechanisms in animal fetuses [5], [29]. We provided visualization of parallel streams in the IVC in the human fetus, including preferential streaming of oxygenated blood from the ductus venosus through the foramen ovale and deoxygenated blood restricted to the right side of the heart. Moreover, radial SVR flow also showed that deoxygenated blood returning from the brain through the SVC is restricted to the right side of the heart and returned to the placenta via the MPA, DA, and DAO. Overall, these observations demonstrated the complex mechanisms in the fetal circulation that shunts oxygenated blood preferentially to the developing brain and heart. These findings support the idea that $3 \mathrm{D}$ visualization of the fetal circulation provides a more complete assessment of fetal circulation and its development than with 2D PCMRI. This technique provides opportunities to study a range of diseases that can place the fetal brain at risk of ischemic-hypoxic injury. For example, it may aid our understanding of various forms of congenital heart disease that impair brain development by altering the streaming of oxygenated blood flow to the fetal brain. Furthermore, by correlating MRI-derived observations of progressively fragmented fetal streaming, clinicians may be able to better plan treatments in-utero and postnatally.

Despite the success of the pipeline, there are limitations to this study. First, fetal radial SVR flow scans were part of a larger clinical research study. As a result, scan time allocated for this component was limited to approximately 6 minutes. This allowed acquisition of only three multislice stacks and without slice overlap, which is less than that used and recommended in similar studies of the fetal brain using SVR reconstruction [13], [16], [32]. This limited the slice coverage. Future work will acquire overlapping slices with larger anatomical coverage to remove the need for inter-slice interpolation. Second, golden-angle radial sampling may suffer from data clustering once data are sorted into cardiac phases
[33]. Clustering can result in large unsampled regions in $k$ space which lead to severe artifacts in the reconstructed images. In future implementations, different sampling patterns will be investigated to counteract this issue.

\section{CONCLUSION}

In conclusion, we have developed an approach for dynamic volumetric fetal flow imaging using highly accelerated multidimensional radial PCMRI. The method makes use of real-time reconstructions for intra-slice motion correction and derivation of fetal heart rate to provide flow sensitive CINEs. They are then combined with inter-slice spatiotemporal registration and volumetric update to create 4D flow reconstructions in human fetuses. This work resulted in the visualizations of complex fetal cardiovascular physiology, such as parallel streaming in the proximal IVC and preferential routes taken by oxygenated blood in the fetal heart. It provides an important means of assessing fetal circulatory system and may enable new ways to manage fetal pathologies.

\section{REFERENCES}

[1] A. Rudolph, Congenital Diseases of the Heart: Clinical-Physiological Considerations. John Wiley \& Sons, 2011.

[2] L. Sun, C. K. Macgowan, J. G. Sled, S.-J. Yoo, C. Manlhiot, P. Porayette, L. Grosse-Wortmann, E. Jaeggi, B. W. McCrindle, J. Kingdom, E. Hickey, S. Miller, and M. Seed, "Reduced Fetal Cerebral Oxygen Consumption is Associated With Smaller Brain Size in Fetuses With Congenital Heart Disease," Circulation, vol. 131, no. 15, pp. 1313-1323, Apr. 2015.

[3] A. Jensen and R. Berger, "Fetal circulatory responses to oxygen lack," J. Dev. Physiol., vol. 16, no. 4, pp. 181-207, Oct. 1991.

[4] A. M. Rudolph and M. A. Heymann, "The circulation of the fetus in utero. Methods for studying distribution of blood flow, cardiac output and organ blood flow," Circ. Res., vol. 21, no. 2, pp. 163-184, Aug. 1967.

[5] D. I. Edelstone and A. M. Rudolph, "Preferential streaming of ductus venosus blood to the brain and heart in fetal lambs," Am. J. Physiol., vol. 237, no. 6, pp. H724-729, Dec. 1979.

[6] M. T. Donofrio, A. J. duPlessis, and C. Limperopoulos, "Impact of congenital heart disease on fetal brain development and injury," Current Opinion in Pediatrics, vol. 23, no. 5, pp. 502-511, Oct. 2011.

[7] F.-T. Lee, M. Seed, L. Sun, and D. Marini, Fetal brain issues in congenital heart disease. 2020.

[8] M. Seed, J. F P van Amerom, S.-J. Yoo, B. Al Nafisi, L. GrosseWortmann, E. Jaeggi, M. S. Jansz, and C. K. Macgowan, "Feasibility of quantification of the distribution of blood flow in the normal human fetal circulation using CMR: a cross-sectional study," Journal of Cardiovascular Magnetic Resonance, vol. 14, no. 1, p. 79, Nov. 2012.

[9] M. Prsa, L. Sun, J. van Amerom, S.-J. Yoo, L. Grosse-Wortmann, E. Jaeggi, C. Macgowan, and M. Seed, "Reference ranges of blood flow in the major vessels of the normal human fetal circulation at term by phase-contrast magnetic resonance imaging," Circ Cardiovasc Imaging, vol. 7, no. 4, pp. 663-670, Jul. 2014.

[10] M. Y. Zhu, N. Milligan, S. Keating, R. Windrim, J. Keunen, V. Thakur, A. Ohman, S. Portnoy, J. G. Sled, E. Kelly, S.-J. Yoo, L. GrossWortmann, E. Jaeggi, C. K. Macgowan, J. C. Kingdom, and M. Seed, "The hemodynamics of late-onset intrauterine growth restriction by MRI," Am. J. Obstet. Gynecol., vol. 214, no. 3, p. 367.e1-367.e17, Mar. 2016.

[11] D. S. Goolaub, C. W. Roy, E. Schrauben, D. Sussman, D. Marini, M. Seed, and C. K. Macgowan, "Multidimensional fetal flow imaging with cardiovascular magnetic resonance: a feasibility study," Journal of Cardiovascular Magnetic Resonance, vol. 20, no. 1, p. 77, Nov. 2018.

[12] L. Feng, R. Grimm, K. T. Block, H. Chandarana, S. Kim, J. Xu, L. Axel, D. K. Sodickson, and R. Otazo, "Golden-angle radial sparse parallel MRI: combination of compressed sensing, parallel imaging, and golden-angle radial sampling for fast and flexible dynamic volumetric MRI," Magn Reson Med, vol. 72, no. 3, pp. 707-717, Sep. 2014. 
[13] M. Kuklisova-Murgasova, G. Quaghebeur, M. A. Rutherford, J. V. Hajnal, and J. A. Schnabel, "Reconstruction of fetal brain MRI with intensity matching and complete outlier removal," Medical Image Analysis, vol. 16, no. 8, pp. 1550-1564, Dec. 2012.

[14] F. Rousseau, O. A. Glenn, B. Iordanova, C. Rodriguez-Carranza, D. B. Vigneron, J. A. Barkovich, and C. Studholme, "Registration-based approach for reconstruction of high-resolution in utero fetal MR brain images," Acad Radiol, vol. 13, no. 9, pp. 1072-1081, Sep. 2006.

[15] B. Kainz, M. Steinberger, W. Wein, M. Kuklisova-Murgasova, C. Malamateniou, K. Keraudren, T. Torsney-Weir, M. Rutherford, P. Aljabar, J. V. Hajnal, and D. Rueckert, "Fast Volume Reconstruction From Motion Corrupted Stacks of 2D Slices," IEEE Trans. Med. Imaging, vol. 34, no. 9, pp. 1901-1913, Sep. 2015.

[16] J. F. P. van Amerom, D. F. A. Lloyd, M. Deprez, A. N. Price, S. J. Malik, K. Pushparajah, M. P. M. van Poppel, M. A. Rutherford, R. Razavi, and J. V. Hajnal, "Fetal whole-heart 4D imaging using motioncorrected multi-planar real-time MRI," Magn Reson Med, vol. 82, no. 3, pp. 1055-1072, 2019.

[17] T. A. Roberts, J. F. P. van Amerom, A. Uus, D. F. A. Lloyd, M. P. M. van Poppel, A. N. Price, J.-D. Tournier, C. A. Mohanadass, L. H. Jackson, S. J. Malik, K. Pushparajah, M. A. Rutherford, R. Rezavi, M. Deprez, and J. V. Hajnal, "Fetal whole heart blood flow imaging using 4D cine MRI," Nature Communications, vol. 11, no. 1, p. 4992, Oct. 2020.

[18] M. Lustig, D. Donoho, and J. M. Pauly, "Sparse MRI: The application of compressed sensing for rapid MR imaging," Magn Reson Med, vol. 58, no. 6, pp. 1182-1195, Dec. 2007.

[19] M. S. Jansz, M. Seed, J. F. P. van Amerom, D. Wong, L. GrosseWortmann, S.-J. Yoo, and C. K. Macgowan, "Metric optimized gating for fetal cardiac MRI," Magn Reson Med, vol. 64, no. 5, pp. 1304-1314, Nov. 2010.

[20] P. G. Walker, G. B. Cranney, M. B. Scheidegger, G. Waseleski, G. M Pohost, and A. P. Yoganathan, "Semiautomated method for noise reduction and background phase error correction in MR phase velocity data," Journal of Magnetic Resonance Imaging, vol. 3, no. 3, pp. 521530, 1993.

[21] S. P. Borgatti and M. G. Everett, "Models of core/periphery structures," Social Networks, vol. 21, no. 4, pp. 375-395, Oct. 2000.

[22] E. M. Schrauben, J. M. Lim, D. S. Goolaub, D. Marini, M. Seed, and C. K. Macgowan, "Motion robust respiratory-resolved 3D radial flow MRI and its application in neonatal congenital heart disease," Magn Reson Med, vol. 83, no. 2, pp. 535-548, 2020.

[23] C. W. Roy, M. Seed, J. C. Kingdom, and C. K. Macgowan, "Motion compensated cine CMR of the fetal heart using radial undersampling and compressed sensing," Journal of Cardiovascular Magnetic Resonance, vol. 19, no. 1, p. 29, Mar. 2017.

[24] M. A. Gulsun, M.-P. Jolly, J. Guehring, C. Guetter, A. Littmann, A. Greiser, M. Markl, and A. F. Stalder, "A Novel 4D Flow Tool for Comprehensive Blood Flow Analysis," p. 1.

[25] S. Yagel, N. H. Silverman, and U. Gembruch, Fetal cardiology: embryology, genetics, physiology, echocardiographic evaluation, diagnosis, and perinatal management of cardiac diseases. CRC Press, 2018.

[26] K. Mukai, N. S. Burris, V. S. Mahadevan, E. D. Foster, K. G. Ordovas, and M. D. Hope, "4D flow image quality with blood pool contrast: a comparison of gadofosveset trisodium and ferumoxytol," Int $J$ Cardiovasc Imaging, vol. 34, no. 2, pp. 273-279, Feb. 2018.

[27] S. S. Vasanawala, K.-L. Nguyen, M. D. Hope, M. D. Bridges, T. A. Hope, S. B. Reeder, and M. R. Bashir, "Safety and Technique of Ferumoxytol Administration for MRI," Magn Reson Med, vol. 75, no. 5, pp. 2107-2111, May 2016.

[28] H. S. Thomsen, S. K. Morcos, and P. Dawson, "Is there a causal relation between the administration of gadolinium based contrast media and the development of nephrogenic systemic fibrosis (NSF)?," Clin Radiol, vol. 61, no. 11, pp. 905-906, Nov. 2006.

[29] E. M. Schrauben, B. S. Saini, J. R. T. Darby, J. Y. Soo, M. C. Lock, E. Stirrat, G. Stortz, J. G. Sled, J. L. Morrison, M. Seed, and C. K. Macgowan, "Fetal hemodynamics and cardiac streaming assessed by 4D flow cardiovascular magnetic resonance in fetal sheep," Journal of Cardiovascular Magnetic Resonance, vol. 21, no. 1, p. 8, Jan. 2019.

[30] J. A. Macdonald, P. A. Corrado, S. M. Nguyen, K. M. Johnson, C. J. Francois, R. R. Magness, D. M. Shah, T. G. Golos, and O. Wieben, "Uteroplacental and Fetal 4D Flow MRI in the Pregnant Rhesus
Macaque," J Magn Reson Imaging, vol. 49, no. 2, pp. 534-545, Feb. 2019.

[31] E. M. Schrauben, J. R. T. Darby, B. S. Saini, S. L. Holman, M. C. Lock, S. R. Perumal, M. Seed, J. L. Morrison, and C. K. Macgowan, "Technique for comprehensive fetal hepatic blood flow assessment in sheep using 4D flow MRI," J. Physiol. (Lond.), vol. 598, no. 17, pp. 3555-3567, Sep. 2020.

[32] A. Gholipour, O. Afacan, I. Aganj, B. Scherrer, S. P. Prabhu, M. Sahin, and S. K. Warfield, "Super-resolution reconstruction in frequency, image, and wavelet domains to reduce through-plane partial voluming in MRI," Med Phys, vol. 42, no. 12, pp. 6919-6932, Dec. 2015.

[33] F. Han, Z. Zhou, S. Rapacchi, K.-L. Nguyen, J. P. Finn, and P. Hu, "Segmented golden ratio radial reordering with variable temporal resolution for dynamic cardiac MRI: Segmented Golden Ratio Radial Reordering for Cardiac MRI," Magn. Reson. Med., vol. 76, no. 1, pp. 94-103, Jul. 2016. 